\title{
Split Unilateral del Nervio Femoral a Nivel del Músculo Psoas Mayor: Reporte de Caso
}

\author{
Unilateral Femoral Nerve Split at the Psoas Major Muscle: Case Report
}

\author{
Ana Barriga ${ }^{1}$; Gerardo Méndez ${ }^{1,2}$ \& Patricia Hernandez ${ }^{1}$
}

\begin{abstract}
BARRIGA, A.; MÉNDEZ, G. \& HERNANDEZ, P. Split unilateral del nervio femoral a nivel del músculo psoas mayor: reporte de caso. Int. J. Morphol., 39(6):1673-1676, 2021.

RESUMEN: El nervio femoral (NF) es el mayor o ramo del plexo lumbar. Normalmente se origina de las divisiones posteriores del segundo al cuarto ramo anterior del plexo lumbar (L2-L4). El músculo psoas mayor tiene su origen a nivel de las vértebras T12 a L5, se fusiona con el músculo ilíaco para luego insertarse en el trocánter menor del fémur. Normalmente, a nivel de la pelvis menor el NF se encuentra entre los músculos ilíaco y psoas mayor. En este trabajo presentamos un caso donde el músculo psoas mayor se relaciona con divisiones o split del NF, esta es una rara variación en la división y curso del NF con relación al músculo psoas mayor. Se observó que el NF se dividía en dos ramos por sobre el plano del ligamento inguinal después de su origen en el plexo lumbar. El NF del lado izquierdo se formó por las ramas ventrales de L2 a L4, a nivel de L5 el nervio es perforado por fascículos del músculo psoas mayor. La división inferior del NF pasaba profundamente a las fibras del músculo iliopsoas y la división superior pasaba superficialmente al músculo psoas mayor y profundo a la fascia ilíaca. Después de un trayecto de 60,21 mm ambas divisiones se unieron, después de atrapar fibras músculo iliopsoas justo inmediatamente proximal al ligamento inguinal para formar el tronco del NF. Si bien las causas embriológicas de las variaciones de los nervios periféricos se remontan a la quinta y sexta semana de vida intrauterina, la expresión clínica de disfunciones neuromusculares aparecerá varios decenios después. De modo que los médicos de las áreas de la traumatología y neurología deben estar al tanto de tales variantes anatómicas para entender mejor el dolor y los síndromes asociados a la compresión nerviosa y durante las maniobras quirúrgicas en esta región.
\end{abstract}

PALABRAS CLAVE: Nervio femoral; Musculo psoas; Neuroanatomía.

\section{INTRODUCCIÓN}

El dolor del miembro inferior producto de una hernia del disco lumbar es una situación clínica común. Sin embargo, ciertas variantes musculares y de nervios periféricos pueden generar una sintomatología similar y representan una etiología no reconocida de la disfunción del nervio femoral (NF). Esos casos pueden afectar el resultado de esquemas terapéuticos específicos. Por lo tanto, el reconocimiento de esas variaciones anatómicas puede ser de utilidad para el clínico cuando trate a un paciente con dolor del miembro inferior con sintomatología recurrente. En algunos informes se han notificado variaciones anatómicas en los músculos psoas e ilíaco, asociados a una división (split) del NF causando un posible riesgo de atrapamiento de éste (Khalid et al., 2017).

El músculo psoas mayor presenta un aspecto fusiforme y se encuentra dispuesto a los lados de la región lumbar, relacionándose estrechamente con los procesos transversos de las vértebras lumbares. El músculo psoas mayor se une al músculo ilíaco para formar el músculo iliopsoas. Como parte del músculo iliopsoas, el músculo psoas mayor contribuye en gran medida a la flexión de la articulación de la cadera (Khalid et al.). El músculo psoas mayor se origina en cara anterolateral del cuerpo de las vértebras T12 a L5, en los discos intervertebrales relacionados y en los procesos transversos de las vértebras lumbares, entre otros lugares, pasa por posterior al ligamento inguinal junto con el músculo ilíaco y se inserta en el trocánter menor del fémur. El NF es una de las ramas más grandes del plexo lumbar y surge de las divisiones dorsales de los ramos ventrales de los nervios espinales L2-L4 (Ashwini et al., 2017; Alveal-Mellado et al., 2019) y ocasionalmente, la rama ventral de L1 y/o L5 contribuye a la formación del NF (Wong et al., 2019). EL NF desciende en relación con el margen lateral del músculo psoas y luego se dispone en el hiato entre este y el músculo

\footnotetext{
${ }^{1}$ Instituto Anatomía, Histología y Patología. Facultad de Medicina. Universidad Austral de Chile.

${ }^{2}$ Escuela de Graduados. Facultad de Medicina. Universidad Austral de Chile, Valdivia, Chile.
} 
ilíaco; contenido en la laguna muscular, aborda el compartimiento anterior del muslo para inervar los músculos del compartimiento y la piel de la zona anterior del muslo, llegando a la parte distal de la pierna e incluso el pie, a través del nervio safeno (Ashwini et al.). Los ramos nerviosos pueden ser atrapados entre los fascículos musculares por donde transitan, situación que puede generar una sintomatología similar a las compresiones de fibras nerviosas entre tejidos duros; sin embargo, la conducta terapéutica para estos casos es diferente. El objetivo de este trabajo es reportar una variación en la división y el curso del nervio femoral izquierdo, cuyas fibras quedan entrelazadas con los fascículos musculares del músculo psoas.

\section{REPORTE DE CASO}

El Split del NF se presentó en un cadáver de un individuo adulto, de sexo masculino, donado con fines docentes por el Departamento de Anatomía Patológica del Hospital Base de Valdivia al Instituto de Anatomía, Histología y Patología de la Facultad de Medicina de la Universidad Aus-

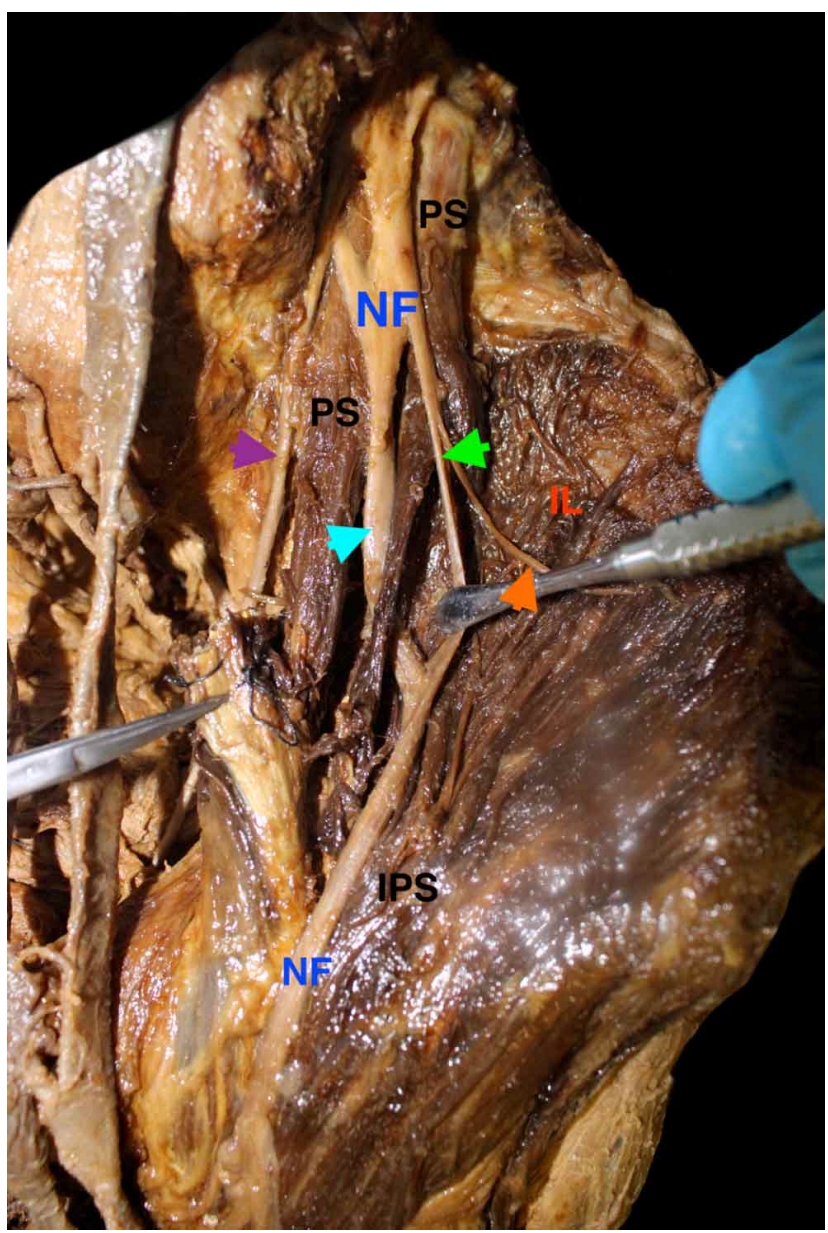

tral de Chile. El cadáver fue fijado con formaldehído al 10 $\%$ diluido en agua destilada. Después de seis meses en esta solución, el cuerpo se lavó y se mantuvo en agua para comenzar la disección. El procedimiento de disección de la pelvis y de las miembros inferiores se realizó acorde a lo descrito en la guía de disección del cuerpo humano de Loukas et al. (2013).

Durante la disección, observamos en el lado izquierdo del cuerpo una rara variación en la división y el curso del nervio femoral y el músculo psoas mayor (Fig. 1). El nervio femoral, después de su origen en el plexo lumbar, a nivel de la fosa ilíaca, presentaba dos divisiones. El NF estaba formado por las ramas ventrales de L2 a L4, y a nivel de L5 se bifurcaba en dos ramas separadas por fibras del músculo psoas. La división inferior del NF pasaba profundamente a las fibras del músculo psoas mayor y la división superior pasaba superficialmente al músculo psoas mayor y profundo a la fascia ilíaca. Después de un trayecto de 60,21 mm, ambas divisiones se unieron atrapando en el ojal nervioso a fibras del músculo ilíaco; justo a nivel del ligamento inguinal ambas ramas se unieron para formar el nervio femoral. El nervio femoral ingreso al muslo, posterior al ligamento inguinal y lateralmente a la vaina femoral y en el triángulo femoral se dividió en sus ramos anteriores y posteriores. Los ramos de ambas divisiones se distribuyeron por los músculos y la piel de la parte anteromedial del muslo. Una pequeña rama del NF (nervio safeno) continuaba su trayecto en dirección a la pierna. En el lado derecho del cadáver, el origen y el trayecto del NF correspondía a la distribución normal descrita por la literatura.

\section{DISCUSIÓN}

Las neuropatías que afectan al NF son poco frecuentes en comparación con otros nervios del miembro inferior (Chen et al., 1995; De Burca, 2011). La neuropatía femoral puede ser el resultado de la compresión directa o indirecta del NF entre el músculo psoas mayor y la pared pélvica. Por otra parte, el músculo iliopsoas, un músculo flexor clave de la articulación de la cadera, se lesiona muy a menudo durante las cirugías que afectan a dicha articulación y a la región lumbar inferior (Ashwini et al.). Jelev et al. (2005) reportaron un caso de división (split) bilateral del NF encon-

Fig. 1. Vista anterior de disección fosa iliaca izquierda. NF: Nervio femoral; PS; músculo psoas mayor izquierdo; IL: músculo iliaco izquierdo; IPS: músculo iliopsoas izquierdo; flecha Cyan: rama profunda del NF; Flecha Verde: rama superficial del NF. flecha Morada: nervio obturador; flecha Naranja: ramo para músculo iliaco. 
trada en la pelvis de una mujer, junto con la variación en su trayecto. Por otro lado, se han descrito variantes anatómicas del músculo iliopsoas (Jelev et al.; Tubbs et al., 2006; Wong et al.). Clarkson \& Rainy (1889) descubrieron una subdivisión inusual del músculo psoas mayor en cuatro fascículos musculares a cada lado durante la disección de un cadáver adulto de sexo masculino y de mediana edad (Clarkson \& Rainy). Durante la disección rutinaria de la pared abdominal posterior de un cadáver femenino caucásico de 71 años, Wong et al. observaron una variante muscular del posas mayor en el lado izquierdo que dividía el NF en múltiples partes similar a lo descrito por (Clarkson \& Rainy). Este músculo se originaba en la superficie anteromedial del cuadrado lumbar y discurría distalmente para fusionarse con el tendón del psoas mayor a nivel del ligamento inguinal. El NF de este lado estaba formado por las ramas ventrales de L2 a L4 a nivel de L4 y se bifurcaba en cuatro ramas separadas a nivel de L5. La rama más medial descendía por detrás de la variante del músculo psoas mayor e inervaba el musculo iliaco. el segundo y tercer ramo se dividían por el músculo variante a lo largo de $101,9 \mathrm{~mm}$, se reunían lateralmente al músculo variante y luego pasaban por debajo del ligamento inguinal. El ramo más lateral corría hacia abajo y anterior al músculo variante, pasaba independientemente por debajo del ligamento inguinal sin reunirse, e inervaba la piel de la cara lateral del muslo junto con el nervio cutáneo femoral lateral. El NF tenía ramas directas que abastecían a los músculos ilíaco, psoas mayor y psoas cuarto. La división den NF también ha sido reportada por una variación del musculo iliaco por datos concuerdan con lo reportado por Ashwini et $a l$. La variación del músculo ilíaco se originaba en la cara medial de la cresta ilíaca y perforaba el nervio femoral en dos partes: la porción más pequeña discurría superficialmente a la variación del musculo iliaco y la porción más grande se encontraba entre la variación y el músculo ilíaco. Estos reportes concuerdan con nuestro estudio en el origen del NF se originó por los ramos ventrales de L2 a L4, luego bifurcarse en la zona de la variante muscular y posteriormente estos ramos se unen en la zona de la fosa iliaca para formar el NF que atraviesa el ligamento inguinal.

El NF puede presentar divisiones en relación a fibras del músculo psoas mayor como describen Vázquez et al. (2007). Estos autores señalaron que en 19 de los 242 especímenes examinados el NF se presentaba dividido por fascículos del músculo psoas mayor o del ilíaco. De esos 19 especímenes, en cuatro muestras el psoas mayor perforaba la NF; en doce especímenes el músculo ilíaco perforaba la NF; en un espécimen el nervio era perforado por fibras de los músculos psoas y del ilíaco; y en dos especímenes el NF estaba cubierto por un fascículo del músculo psoas mayor. Estas variaciones del NF no tuvieron una relación significativa según el sexo o el lado.
Kirchmair et al. (2008) encontraron que el NF presentaba una perforación por fascículos del músculo psoas mayor en nueve (9) de 61 especímenes, y dos perforaciones por fascículos del músculo psoas mayor en cuatro (4) de 61 especímenes. En 11 de 13 especímenes, la reunificación de cada ramo se observó en relación con el margen lateral del músculo psoas mayor próximo al ligamento inguinal, y en dos de 13 casos no hubo reunificación antes de salir de la pelvis. Anloague \& Huijbregts (2009) estudiaron 34 plexos lumbares y revelaron que el 35,3\% de los NF estaban divididos en dos o tres partes por fascículos del músculo psoas mayor. Las variaciones del NF y el músculo psoas mayor podrían afectar los abordajes quirúrgicos de la región, como también el bloqueo del plexo lumbar, y lamanipulación de la arteria femoral para la realización de angiografías u otros procedimientos de la región pélvica (Anloague \& Huijbregts; Kirchmair et al.; Wong et al.).

Hacia la quinta semana de la vida intrauterina los axones motores surgen desde la médula espinal en desarrollo, siguiendo una gradiente cráneo-caudal, siendo los axones motores los que preceden al desarrollo de los axones sensitivos de los nervios raquídeos (Larsen 2003). Los axones motores en crecimiento no emiten colaterales y progresan en busca de sus órganos blanco, los mioblastos, para formar las uniones neuromusculares. Como los axones motores preceden a los axones sensitivos, es posible que algunas fibras musculares se interpongan entre los axones del nervio en desarrollo, determinando la formación de nervios perforados por fascículos musculares (Wong et al.).

Todas las variaciones del NF y el músculo psoas mayor fueron diferentes en cantidad y posición con respecto a nuestro informe. El conocimiento de tales variaciones anatómicas puede explicar la etiología de la compresión o daño del NF, así como al síndrome compartimental posterior a una cirugía en la región lumbar y de la cadera (Tubbs et al.; Wong et al.). Finalmente, el médico cirujano debe considerar estas anomalías musculares y del NF en pacientes con síntomas de compresión del nervio femoral en los que no se encuentra ninguna otra etiología clara. Además, los radiólogos deben tener en cuenta estas posibles anomalías al interpretar la anatomía de esta región asociada a la sintomatología de los pacientes.

\section{AGRADECIMIENTOS}

Queremos agradecer al Dr. Oscar Inzunza de la PUC, por su desinteresada y constante ayuda. Gracias por estar siempre disponible para atender nuestras dudas, colaborar con nuestra Universidad Austral. 
BARRIGA, A.; MÉNDEZ, G. \& HERNANDEZ, P. Unilateral femoral nerve split at the psoas major muscle: case report. Int. J. Morphol., 39(6):1673-1676, 2021.

SUMMARY: AbstractThe femoral nerve (NF) is the major branch (or ramus) of the lumbar plexus. It normally originates from the posterior divisions of the second to fourth anterior branches of the lumbar plexus (L2-L4). The psoas major muscle originates at the level of the T12 to L5 vertebrae, fuses with the iliacus muscle and then inserts into the lesser trochanter of the femur. Normally, at the level of the lesser pelvis, the NF is found between the iliacus and psoas major muscles. In this paper we present a case where the psoas major muscle is related to divisions or splitting of the NF, this is a rare variation in the division and course of the NF in relation to the psoas major muscle. The NF was observed to divide into two branches above the plane of the inguinal ligament after its origin in the lumbar plexus. The NF on the left side was formed by ventral branches from L2 to L4, at the level of L5 the nerve is perforated by fascicles of the psoas major muscle. The lower division of the NF passed deep to the fibers of the iliopsoas muscle and the upper division passed superficial to the psoas major muscle and deep to the iliac fascia. After a path of $60.21 \mathrm{~mm}$ both divisions joined, after trapping iliopsoas muscle fibers just immediately proximal to the inguinal ligament to form the NF trunk. While the embryological causes of peripheral nerve variations date back to the fifth and sixth week of intrauterine life, the clinical expression of neuromuscular dysfunctions will appear several decades later. Thus, physicians in the areas of traumatology and neurology should be aware of such anatomical variants to better understand pain and syndromes associated with nerve compression and during surgical maneuvers in this region.

KEY WORDS: Femoral Nerve [MeSH]; Psoas Muscle $[\mathrm{MeSH}] \&$ Neuroanatomy [MeSH]

\section{REFERENCIAS BIBLIOGRÁFICAS}

Alveal-Mellado, D.; Sousa-Rodrigues, C. F. \& Olave, E. Innervation branches and distribution of the femoral nerve in the quadriceps femoral muscle in Brazilian individuals. Int. J. Morphol., 37(2):712-8, 2019.

Anloague, P. A. \& Huijbregts, P. Anatomical variations of the lumbar plexus: a descriptive anatomy study with proposed clinical implications. J. Man. Manip. Ther., 17(4):e107-14, 2009.

Ashwini, L. S.; Somayaji, S. N.; Rao, M. \& Marpalli, S. Preinguinal splitting and reunion of femoral nerve entrapping the fleshy fibres of iliacus muscle - A case report. J. Clin. Diagn. Res., 11(4):AD01-2, 2017.

Chen, S. S.; Lin, A. T.; Chen, K. K. \& Chang, L. S. Femoral neuropathy after pelvic surgery. Urology, 46(5):575-6, 1995.

Clarkson, R. D. \& Rainy, H. Unusual arrangement of the psoas muscle. $J$. Anat. Physiol., 23(Pt. 3):504-6, 1889.

De Burca, N. Anterior thigh pain - A case report. Man. Ther., 16(3):291-5, 2011.

Jelev, L.; Shivarov, V. \& Surchev, L. Bilateral variations of the psoas major and the iliacus muscles and presence of an undescribed variant muscle-accessory iliopsoas muscle. Ann. Anat., 187(3):281-6, 2005.

Khalid, S.; Iwanaga, J.; Loukas, M. \& Tubbs, R. S. Split femoral nerve due to psoas tertius muscle: a review with other cases of variant muscles traversing the femoral nerve. Cureus, 9(8):e1555, 2017.
Kirchmair, L.; Lirk, P.; Colvin, J.; Mitterschiffthaler, G. \& Moriggl, B. Lumbar plexus and psoas major muscle: not always as expected. Reg. Anesth. Pain Med., 33(2):109-14, 2008.

Loukas, M.; Benninger, B. \& Tubbs, R. S. Gray's Clinical Photographic Dissector of the Human Body. Philadelphia, Elsevier Sauders, 2013.

Tubbs, R. S.; Oakes, W. J. \& Salter, E. G. The psoas quartus muscle. Clin. Anat., 19(7):678-80, 2006.

Vázquez, M. T.; Murillo, J.; Maranillo, E.; Parkin, I. G. \& Sanudo, J. Femoral nerve entrapment: a new insight. Clin. Anat., 20(2):175-9, 2007.

Wong, T. L.; Kikuta, S.; Iwanaga, J. \& Tubbs, R. S. A multiply split femoral nerve and psoas quartus muscle. Anat. Cell Biol., 52(2):208-10, 2019.

Dirección para correspondencia:

Gerardo Méndez

Instituto Anatomía

Histología y Patología

Facultad de Medicina

Universidad Austral de Chile

CHILE

E-mail: Gerardo.mendez@uach.cl

Recibido : 14-06-2021

Aceptado : 18-08-2021 